\title{
AUTOMATED ATTENDANCE ENTRY SYSTEM BY DETECTION AND RECOGNITION OF THE FACE USING OPEN CV
}

\author{
S.HariPrasath \\ Assistant Professor, \\ Electronics and \\ Communication \\ Engineering, \\ Saranathan College \\ Of Engineering, \\ Trichy, India,
}

\author{
S.Bharathan \\ Electronics and \\ Communication \\ Engineering, \\ Saranathan College \\ Of Engineering, \\ Trichy, India,
}

\section{T.Bharathi Raja}

Electronics and

Communication

Engineering,

Saranathan College

Of Engineering,

Trichy, India,

\author{
V.Gopinath \\ Electronics and \\ Communication \\ Engineering, \\ Saranathan College \\ Of Engineering, \\ Trichy, India,
}

R.Kishore

Electronics and
Communication
Engineering,
Saranathan College
Of Engineering
Trichy, India,

\begin{abstract}
In contemporary world, image processing is used in many fields like medical field, pattern recognition, color processing, etc. The vintage attendance methods like manual entry, finger print scanning, iris scanning are time consuming. Therefore we proposed a system which uses face recognition for attendance entry. We use Opencv-python in Raspbian platform to reduce the cost of the system. Haarcascade algorithm is used as feature for detection. LBPH(Local Binary Pattern Histogram) is used for better recognition of human face
\end{abstract}

Keywords-HaarCascade; LBPH; accuracy;

\section{INTRODUCTION}

Conventional way of taking attendance in schools/institutions is by calling of names by teacher, students responding on their roll numbers and putting ' $\mathrm{A}$ ' or ' $\mathrm{P}$ ' on log book accordingly. Biometric recognition has the potential to become an irreplaceable part of many identification systems used for evaluating the performance of those people working within the organization. Face recognition is a technique of biometric recognition. It is considered to be one of the most successful applications of the image analysis and processing, that is the main reason behind the great attention it has been given in the past several years. Here we are using Haar cascade for face detection.

\section{LITERATURE SURVEY}

[1] Umm-e-Laila, Muzammil Ahmad Khan, Muhammad Kashif Shaikh, Syed Annas bin Mazhar, Khalid Mehboob have proposed the work Comparative analysis for a Real Time Face Recognition System Using Raspberry Pi [2017]. In that, the three algorithms LBPH (Local Binary Pattern Histogram), LCA (Linear Component Analysis), Fisher face used in face recognition are compared for speed and accuracy. It is shown that among all the three algorithms LBPH had the better accuracy. The accuracy rate is $90 \%$.

[2] E.Varadharajan, R.Dharani, S.Jeevitha, B.Kavinmathi, S.Hemalatha have proposed a work called Automatic Attendance Management System Using Face Detection [2016]. In that system they replace the manual attendance method by face detection method. Eigenface algorithm is used for comparing the faces. Face detection is difficult in their work.

[3] B. SreeVidya and E.Chandra have proposed a technique Entropy based Local Binary Pattern (ELBP) feature extraction technique of multimodal biometrics as defence mechanism for cloud storage [2017] to overcome the security threats in Cloudcomputing by getting multiple biometric modalities from users. The ELBP features are extracted and matching is carried out using Multiclass Support Vector Machine (MSVM). The system was built using more than one biometric trait which makes the hacker difficult to imitate all the three traits simultaneously.The application of entropy to feature extraction reveals information about the features under consideration. The accuracy achieved is $89 \%$.

[4] R.R.O. Al-Nima, M.A.M. Abdullah, M.T.S. AlKaltakch, S.S. Dlay, W.L. Woo, J.A. Chambers have proposed the technique [4] Finger texture biometric verification exploiting Multi-scale Sobel Angles Local Binary Pattern features and score-based fusion [2017] . A new feature extraction method called Multiscale Sobel Angles Local Binary Pattern (MSALBP) for application in personal verification using biometric Finger Texture(FT)patterns is used. The MSALBP feature extraction method has been examined and compared with 
different Local Binary Pattern(LBP)types. The database consists of two dimensional finger images. An innovative FCFNN fusion method has also been suggested to improve the verification performance. Each finger will be trained separately and they will get its contribution scores. If the summed scores are matched with the predefined database scores the approval is given by the system. The accuracy achieved is $98 \%$.

[5] Ramadan Gad, Muhammad Talha Ahmed A. Abd ElLatif, M. Zorkany ,

Ayman EL-SAYED , Nawal EL- Fishawy , Ghulam Muhammad have proposed the system Iris Recognition Using Multi-Algorithmic Approaches for Cognitive

Internet of things (CIoT) Framework [2018] based on masking technique to localize Iris for authentication using Internet of Things. Delta-mean and multi-algorithm-mean, were developed to extract iris feature vectors. The proposed system used MQTT protocol and iris image are sent as a password instead of text using MQTT message to the server. Multiple iris images must be taken for each client to increase the accuracy of the system. Due to image contrast limitations, the normalized iris image should be enhanced, therefore Histogram equalization is used to treat low contrast and nonuniform illumination caused by the position of light sources. To classify the iris templates, the enrolled sample is compared with the others stored in the database. In this technique Euclidean distance(ED) is used in feature vector matching of numeric values. The accuracy achieved is $99.49 \%$.

[6] Bin Li, Guang Huo [2016] have proposed the system Face Recognition Using Locality Sensitive Histogram of Oriented Gradients for face recognition which overcomes Histograms of oriented gradients. LSHOG was used to extract feature vectors of face images. LSHOG computes a HOG orientation over the whole images at each pixel loaction. Several experiments were conducted on three benchmark face databases which showed up LSHOG is robust and have achieved satisfactory performance.

[7] Naphtali Abudarsham, Lior Shkiller, Galit Yovel have conducted the experiment Critical Features for Face Recognition [2019] to find the featrures which plays a critical role in face recognition.From that experiment it is found that high PS(Perceptual Sensitivity) plays a major role.Features like eye color,hair color were critical for cauc asian male fgaces which shows up small variation.The features mentioned can be learned through rich experience with familiar faces to obtain optimal generalisation and discrimination.

[8] Yu-Hsuan Tsai, Yih-Cherng Lee, Jian-Jiun Ding, Ronald Y. Chang, and Ming-Chen Hsu have proposed the paper Robust In-Plane and Out-of-Plane Face Detection Algorithm Using Frontal Face Detector and Symmetry Extension [2018] .In that paper, they used to detecting inplane and out-of-plane rotated faces simultaneously is still a challenging task today. A very robust multi-view face detection algorithm, with its core functionality based on frontal face detection, is proposed to simultaneously detect in-plane and out-of-plane rotated faces. Moreover, only the training data in the frontal face is needed and we do not require the training data from different in-plane or out-of-plane rotation angles. In the proposed algorithm, first, techniques such as the skin filter and entropy rate superpixels (ERSs) are applied to obtain face candidates.Then, angle compensation and refinement are applied to improve the accuracy of face detection in the in-plane case. Robust color face detection algorithm incorporating techniques such as the skin filter, ERS superpixel, angle compensation, histogram equalization, validation mechanism, non-maximum suppression, and symmetry extension are proposed. In that proposed algorithm does not required the additional training images with different angles of view; instead, only the training data from the frontal face case and only one frontal face detector are required.

[9] Choon-chin ng ,Mai hoon yap ,Yi- tsang chang,Geesern hsu have proposed the project Hybrid Ageing Pattern For Face Age Estimation [2017] to estimate the age of human being using face detection. In that paper they introduced a Multi-scale wrinkle patterns(MWP) representation, and they investigated the effect of wrinkles on face age estimation and proposed Hybrid Ageing pattern (HAP) for face age estimation .they used set of automatically located facial marks to define the wrinkle region more precisely. The performance of the algorithm is accessed by using mean absolute error on the three state of the art datasets FG-NET,FERET, and MORPH.finally,they propsed HAP which combines the MWP and FAM(facial appearance model).

[10] Khaled Mohammed, A.S. Tolba Mohammed Elmogy have proposed a system called Multimodel Student Attendance System [2018]. In that system they combined both RF ID and face detection and recognition for recognition of student. The student's tag IDs are compared with the student-tag database and then his/her face is detected and recognized using LBPH (Local Binary Pattern Histogram) as feature. The system is complex and RF ID systems are subjected to problems like reader collision, tag collision and high cost. The achieved accuracy is $99 \%$.

[11] Shonal Chaudhry, Rohitash Chandra have implemented the system Face Detection and Recognition in an Unconstrained Environment for Mobile Visual Assistive System [2016]. In that system they used mobile for detection and recognition in which CNN (Convolutional Neural Networks) and classifiers were used. The highest performance achieved is $96.64 \%$ in daylight and $80 \%$ in poor lightning conditions.

[12] Diajeng Tyas Purwa Hapsari, Cindykia Gusti Berliana, Putri Winda, Dr. M.Arief Soeleman have proposed a work called Face Detection Using Haar Cascade in Difference Illumination [2018]. Haarcascade classifier is used to detect the faces by applying features one by one as a group. They tested the system in sunlight 
and lamp conditions individually. It is observed that face detection can work well when using bulbs.

[13]Saikrishna Chatrati, Sumanth Naidu, Ch.Raghava Prasad have implemented the system RFID based Student Monitoring and Attendance Tracking System [2013]. In which, as students enter a classroom, the antenna of a reader placed on the ceiling of classroom would interact with Radio Frequency Identification ("RFID") tags that are worn orcarried by the students. The system would then track which students have entered the classroom, and by comparing the list of entering students with the class list, the system could generate a provisional list of absent students. This system may give rise to the problem of fraudulent access. An unauthorized person may make use of authorized ID card and enter into the organization.

[14] Fahad-Bin-Mazhar, Oli Ahamed, Md.Rasedujjaman have proposed Biometric Smart Attendance Kit with Fingerprint

Scanner by Using Microcontroller. In which An intelligent system based on fingerprint scanner have been designed and implemented that supplanted the traditional mundane attendance system. The system can acquire, store, crosscheck the fingerprint of individuals and export the data in the form of Microsoft excel spreadsheet in a memory module. The entire procedures are supervised by a microcontroller. This system will malfunction when a person makes an entry with wounded thumb.

\section{METHODOLOGY}

The web camera is used to capture the image which consists of group of persons. That captured image is sent to raspberry pi. Raspberry pi is a small size computer with a Broadcom BCM2835 system-on-chip (SoC) multimedia processor. Raspberry pi is connected with the web camera and display device. Within the raspberry pi, Each student face in group image is detected and features are extracted. The SD card is used to store the database of the class students. In order to get perfect match, different images of same student is captured and stored. This will be done for every student in the class. The captured images are then trained by LBPH algorithm. Extracted face is compared with database. Corresponding attendance is displayed.

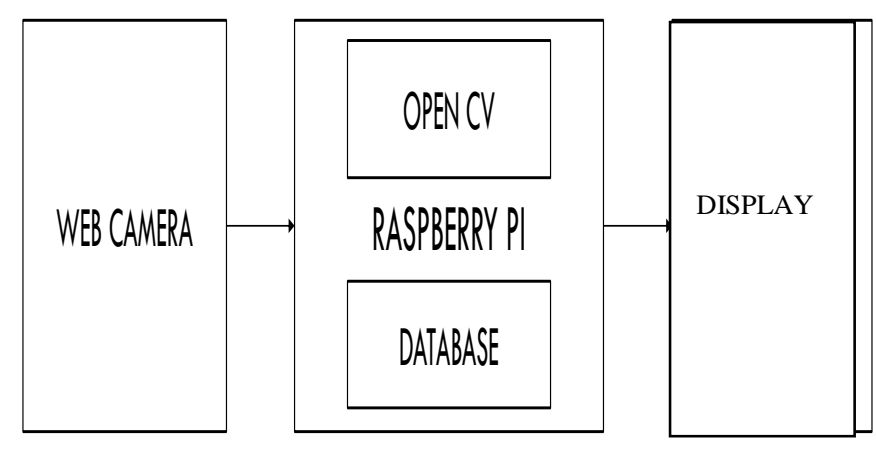

Fig. I Block Diagram

\section{IMPLEMENTATION}

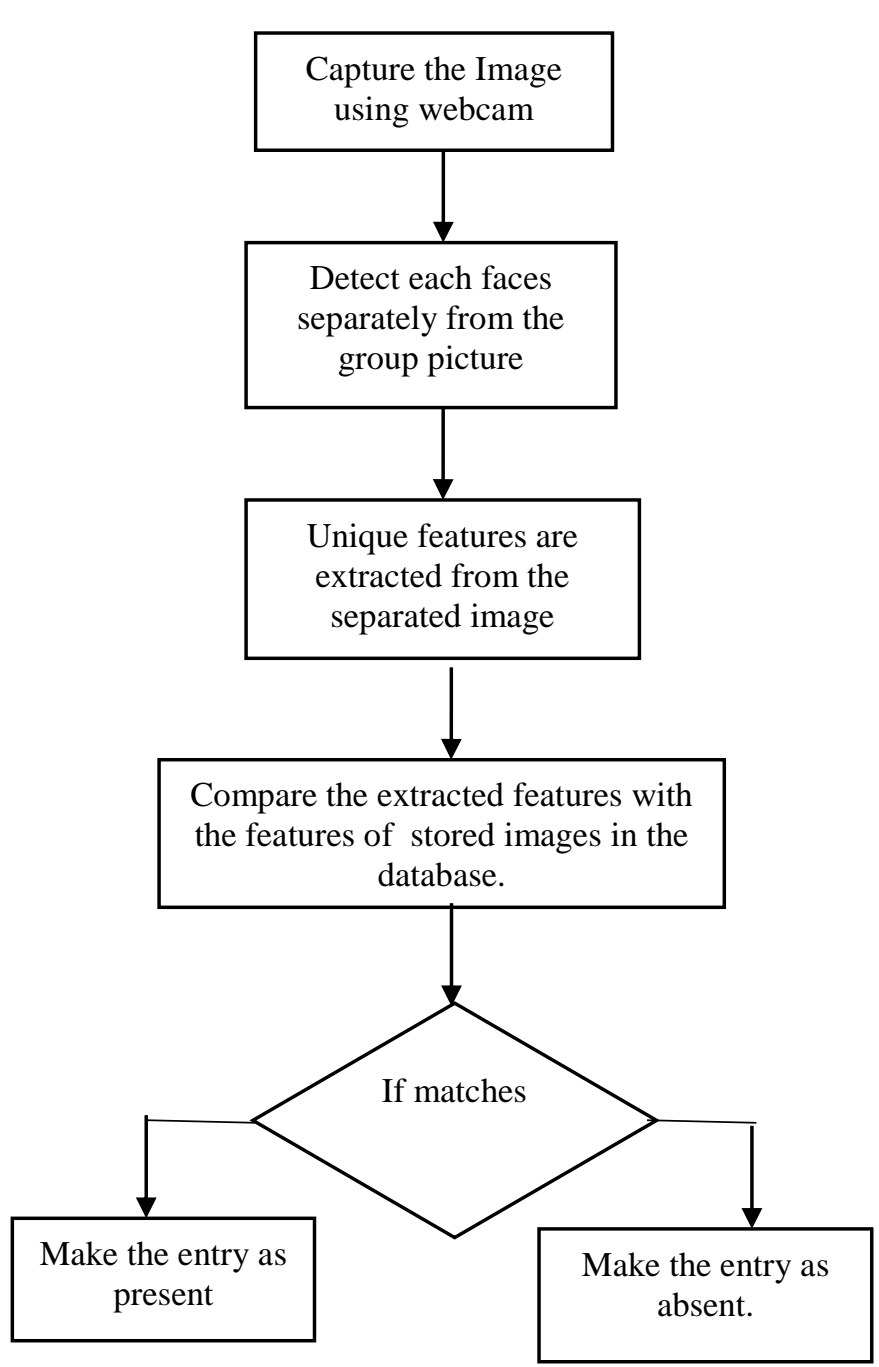

Fig. II Flow Chart

Using webcam group image is captured and each person face is dectected with bouding box using Haar casacade algorithm [A].Haar-like features are digital image features used in object recognition. It is a machine learning based approach where a cascade function is trained from a lot of positive and negative images. It is then used to detect objects in other images. Here we use haarcascade_frontalface algorithm which detects the frontface of human. We need to train the algorithm first. For this we need to use a dataset with the facial images of the people we want to recognize which is shown in Fig. III. Required number of sample images are taken for individual face. Here we use 50 sample images per individual. To train and compare the faces with dataset using LBPH algorithm [B]. While training, the sample 


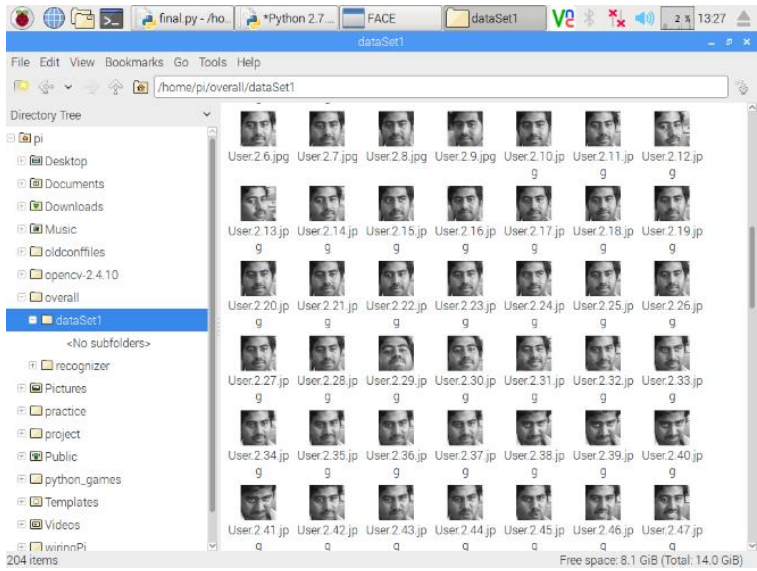

Fig. III dataset

color images are converted into grey scale images and stored in a folder named as "dataSet". At the end of this procedure (LBPH procedure), we have a new image which represents better the characteristics of the original image. To find the image that matches the input image it just need to compare two histograms and return the image with the closest histogram as shown in Fig. IV and V. Corresponding attendance result with time will be displayed in python shell.

The unknown face is displayed with "None" and corresponding entry in python shell will be displayed as "unknown" shown in Fig. VI.

\section{ALGORITHMS}

\section{[A] HAARCASCADE}

Haarcascade algorithm is introduced by Viola and Jones. It is a machine learning method. It is an effective method in which a cascade function is trained from lot of positive and negative images. Haar means a mathematical function that refers rectangle. To train the classifier we need face (positive) images and non face (negative) images. Then features are need to be extracted. They are single values which can be obtained by subtracting sum of pixels in white rectangle from sum of pixels in black rectangle.

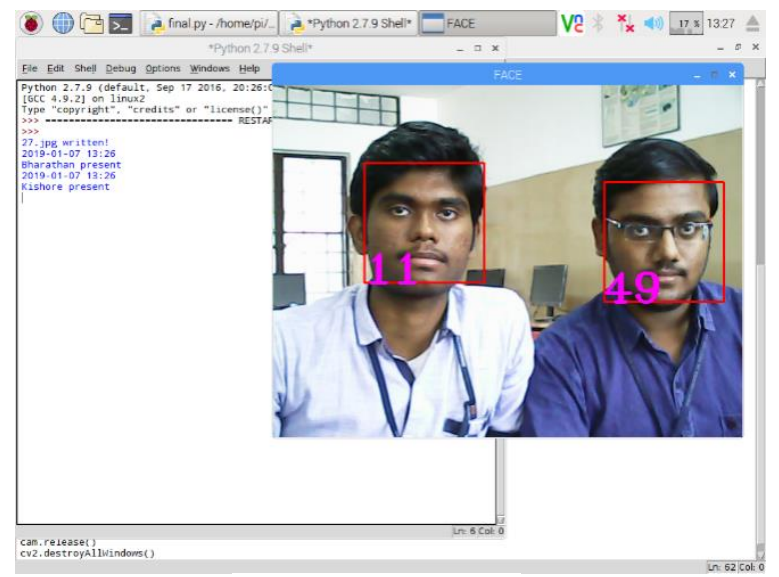

Fig. IV Known faces

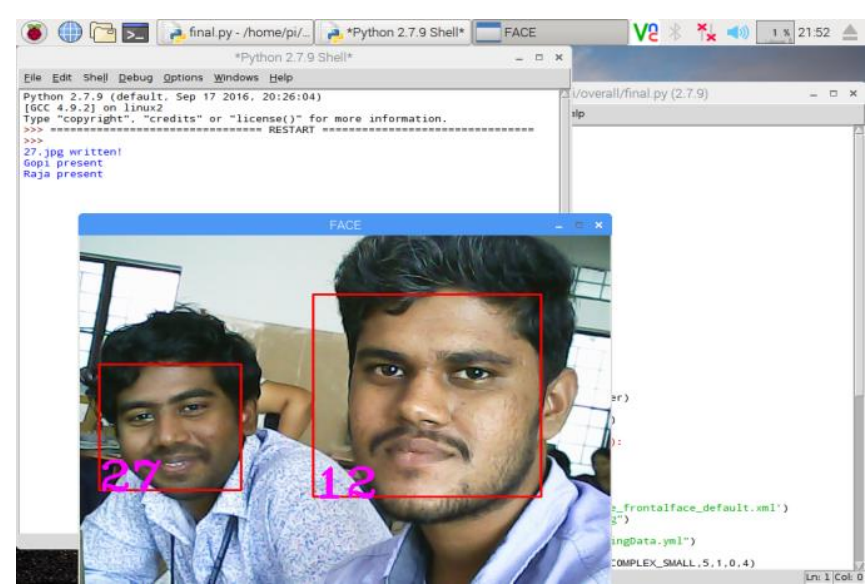

Fig. V Known Faces

Some features are shown in Fig. VII and IX. Edge feature shows the property that the region of eyes is darker than the region of nose and cheeks. Line feature shows the property that eye region is darker than bridgr of nose. Similarlay there are more number of features to extract. Adaboost technique is used to boost the performance of haarcascade algorithm. Adaboost means Adaptive Boosting. It combines multiple weak classifiers to form a strong classifier. Resultant classifier is weighted sum of weak classifiers. So number of features are very much reduced by using this technique. But latency is not reduced. Because thousands of features are to be checked. There are non face regions present more than a face region in an image window. So it is better to have a simple method to decide whether the image has a face or not. So that we can find more time to check possibilities of face region.

For that Cascade of Classifier technique is used. Instead of applying all features, features are grouped. And they are applied one by one. The starting stages contain less number of features. Then the number of features increases in next consecutive stages. If the image window fails the first stage, the process is stopped and the image is identified as negative image. We don't apply next feature stages to that non face image. If the image passes in first

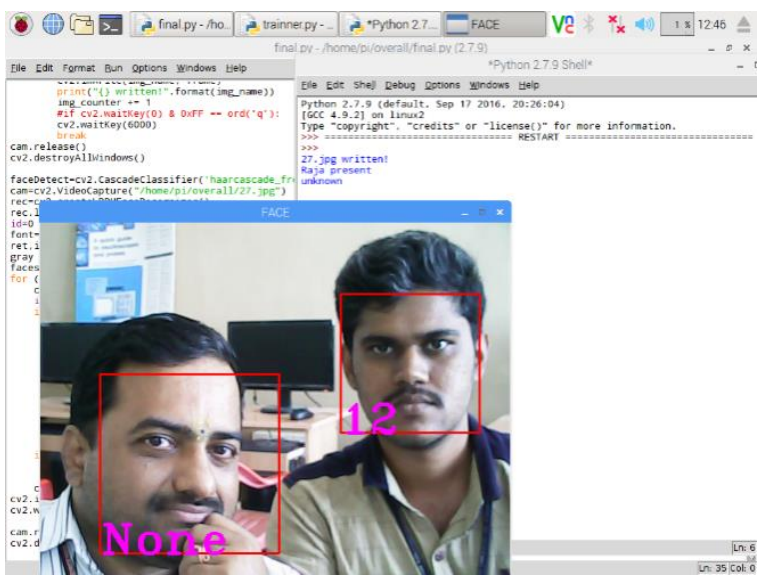

Fig. VI Unknown Face 

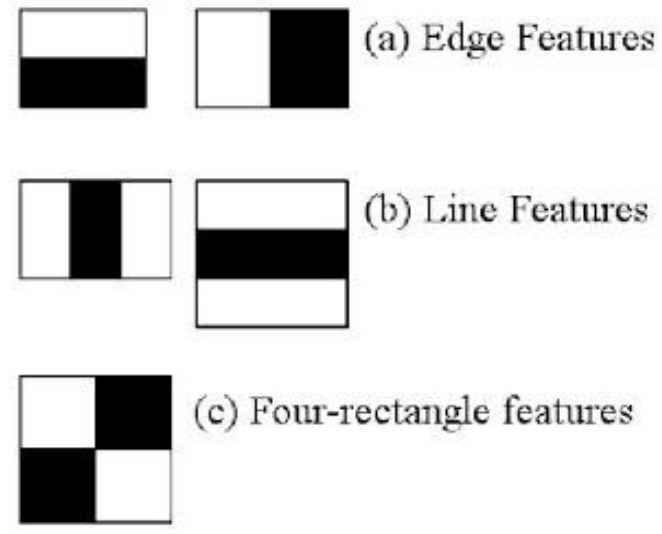

(c) Four-rectangle features

Fig.VII Features

stage, second stage features are applied and the process is continued. Line and Edge features are said to be best two features.

Four Stages of Haarcascade Algorithm

1) Haar Feature Selection,

2) Creating Integral Images,

3) Adaboost Training,

4) Cascading Classifiers.

\section{1) Haar Feature Selection}

First step is to collect haar feature from a image. This is sum of pixel intensities in each region and difference between them in a rectangular window.

\section{2) Creating Integral Images}

It is intermediate representation of image used to compute the rectangle features very rapidly. It can be computed as 4 array references (in Fig. VIII). The value of integral image at location 1 is the sum of pixel intensities in rectangle A.

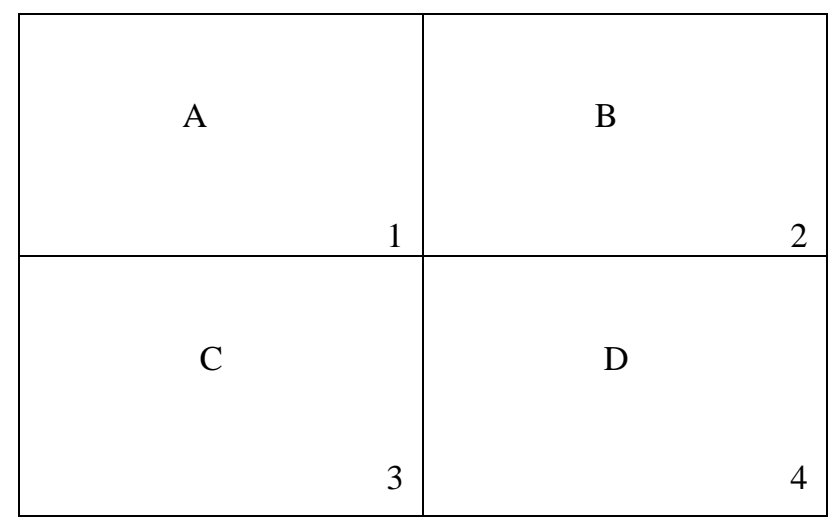

Fig VIII. Array References

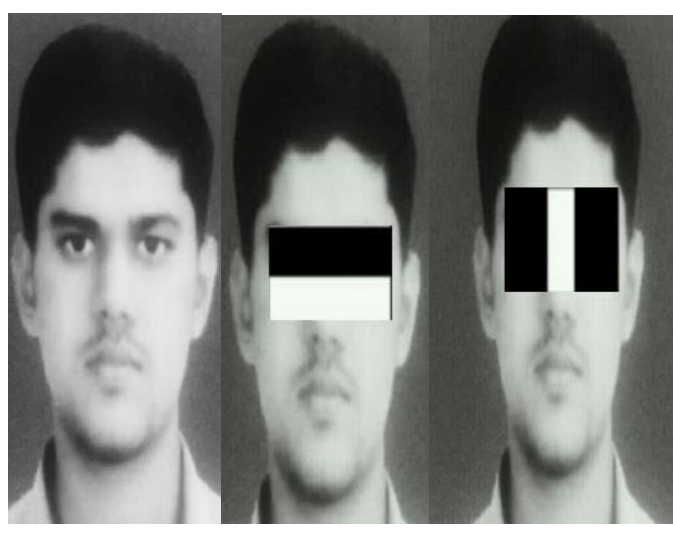

Fig. IX Features

In 2 is $A+B$. In 3 is $A+C$. In 4 is $A+B+C+D$. The sum within $\mathrm{D}$ is $4+1-(2+3)$.

\section{3) Adaboost Training}

It selects only the important features. It reduces dimensionality. So irrelevant features are not calculated. It improves the execution time.

\section{4) Cascading Classifiers}

It has collection of stages. Stages are used to reject negative samples as fast as possible. Finally image can be detected. It can give 3 results. They are TA, FR, FA which are explained in confusion table(Fig XII).

\section{[B] $L B P H$}

Local Binary Pattern (LBP) is a simple yet very efficient texture operator which labels the pixels of an image by thresholding the neighborhood of each pixel and considers the result as a binary number. It was first described in 1994 (LBP) and has since been found to be a powerful feature for texture classification. It has further been determined that when LBP is combined with histograms of oriented gradients (HOG) descriptor, it improves the detection performance considerably on some datasets. Using the LBP combined with histograms we can represent the face images with a simple data vector

The LBPH uses 4 parameters:

1. Radius: the radius is used to build the circular local binary pattern and represents the radius around the central pixel. It is usually set to 1 . 
2. Neighbors: the number of sample points to build the circular local binary pattern.It is usually set to 8 .

3. Grid $\boldsymbol{X}$ : the number of cells in the horizontal direction. The more cells, the finer the grid, the higher the dimensionality of the resulting feature vector. It is usually set to 8 .

4. Grid $Y$ : the number of cells in the vertical direction. The more cells, the finer the grid, the higher the dimensionality of the resulting feature vector. It is usually set to 8 .

First, we need to train the algorithm. To do so, we need to use a dataset with the facial images of the people we want to recognize. We need to also set an ID for each image, so the algorithm will use this information to recognize an input image and give you an output. Images of the same person must have the same ID. The first computational step of the LBPH is to create an intermediate image that describes the original image in a better way, by highlighting the facial characteristics. Suppose we have a facial image in grayscale, We can get part of this image as a window of $3 \times 3$ pixels. It can also be represented as a $3 \times 3$ matrix containing the intensity of each pixel (0 255). Then, we need to take the central value of the matrix to be used as the threshold. This value will be used to define the new values from the 8 neighbors. For each neighbor of the central value (threshold), we set a new binary value. We set 1 for values equal or higher than the threshold and 0 for values lower than the threshold. Now, the matrix will contain only binary values (ignoring the central value). We need to concatenate each binary value from each position from the matrix line by line into a new binary value. Then, we convert this binary value to a decimal value and set it to the central value of the matrix, which is actually a pixel from the original image as shown in Fig. $\mathbf{X}$.

At the end of this procedure (LBP procedure), we have a new image which represents better the characteristics of the original image. Now, using the image generated in the last step, we can use the Grid $\mathbf{X}$ and Grid $\mathbf{Y}$

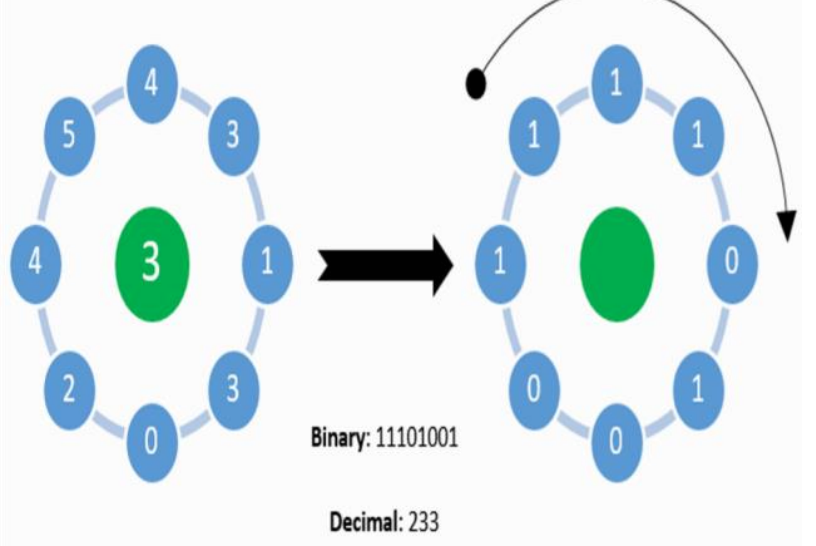

Fig X

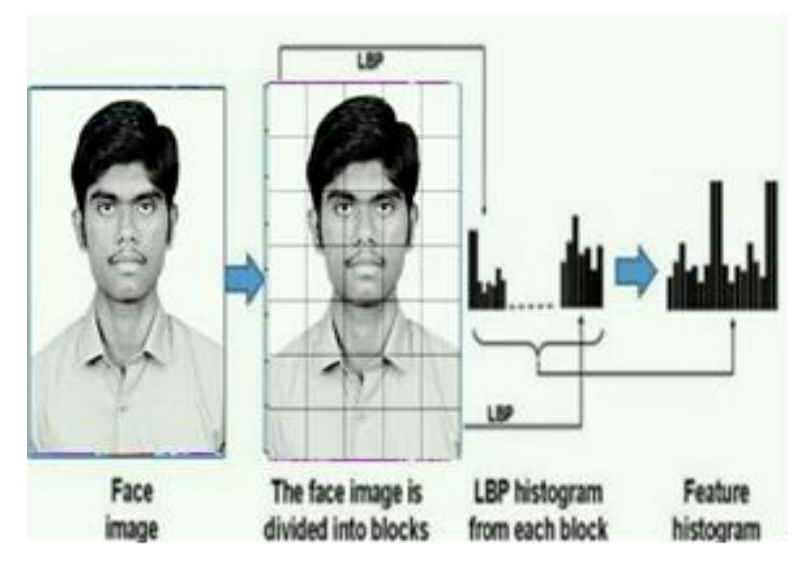

Fig XI

parameters to divide the image into multiple grids. As we have an image in grayscale, each histogram (from each grid) will contain only 256 positions (0 255) representing the occurrences of each pixel intensity. Then, we need to concatenate each histogram to create a new and bigger histogram as shown in Fig. XI.

The final histogram represents the characteristics of the image original image. Each histogram created is used to represent each image from the training dataset. So to find the image that matches the input image we just need to compare two histograms and return the image with the closest histogram.

\section{CONFUSION TABLE}

\begin{tabular}{|c|c|c|c|c|}
\hline ID & 1 & 2 & 3 & 4 \\
\hline 1 & $91 / 100$ & $4 / 100$ & $2 / 100$ & $3 / 100$ \\
\hline 2 & $2 / 100$ & $92 / 100$ & $0 / 100$ & $6 / 100$ \\
\hline 3 & $2 / 100$ & $0 / 100$ & $95 / 100$ & $3 / 100$ \\
\hline 4 & $5 / 100$ & $2 / 100$ & $4 / 100$ & $89 / 100$ \\
\hline
\end{tabular}

Fig. XII Confusion Table 
Confusion table consists three metrics. They are true acceptance (TA), false rejection (FR), false acceptance (FA). TA is the number of times the face image identified correctly. FR is the number of times correct face image is rejected said that image belong to someone's image. FA is the number of times other person face image is identified as current face image. Confusion table for four students of our work is shown in Fig XII.

\section{RESULT}

Using this method we can replace all the old methods. Efficient and automatic attendance management is introduced in paper. This method requires only simple hardware for installation. The management of attendance in this method is more simple and the attendance is taken more accurately. If the person face is not in perfect position, the output will be incorrect. Dim light affects the system performance, therefore it cannot detect the image completely. we are working on it. We achieved the system accuracy as $91.75 \%$.

\section{REFERENCE}

[1] Muzammil ahmad khan,Muhammad kashikh,syed annas bin mazhar, "Comparative analysis for a real time face recognition system using raspberry pi” Nov 2017 IEEE.

[2] E.Varadharajan, R.Dharani, jeevitha, B,kavinmathi, S.Hemalatha, "Automatic Attendance Management System using Face Detection”,Sep 2016 IEEE.

[3] B. SreeVidya and E.Chandra, "Entropy based Local Binary Pattern (ELBP) Feature Extraction Technique of Multimodal Biometrics as Defence Mechanism for Cloud Storage" [2017].

[4] R.R.O. Al-Nima, M.A.M. Abdullah, M.T.S. Al-

Kaltakch, S.S. Dlay, W.L. Woo, J.A. Chambers, "Finger texture biometric verification exploiting Multi-scale Sobel Angles Local Binary Pattern features and score-based fusion “[2017].
[5] Ramadan Gad, Muhammad Talha, Ahmed A. Abd ElLatif , M. Zorkany,

Ayman EL-SAYED, Nawal EL- Fishawy , Ghulam Muhammad, "Iris Recognition Using Multi-Algorithmic Approaches for Cognitive Internet of things (CIoT) Framework" [2018].

[6] Bin Li, Guang Huo "Face Recognition Using Locality Sensitive Histogram of Oriented Gradients" [2016].

[7] Naphtali Abudarsham, Lior Shkiller,

"Critical Features for Face Recognition" [2019].

[8] Yu-Hsuan Tsai, Yih-Cherng Lee, Jian-Jiun Ding , Ronald Y. Chang, and Ming-Chen Hsu, "Robust In-Plane and Out-of-Plane Face Detection Algorithm Using Frontal Face Detector and Symmetry Extension" [2018].

[9] Choon-chin ng ,Mai hoon yap ,Yi- tsang chang ,Geesern hsu, "Hybrid Ageing Pattern For Face Age Estimation" [2017].

[10] Khaled Mohammed, A.S. Tolba Mohammed Elmogy, "Multimodel Student Attendance System" [2018].

[11] Shonal Chaudhry, Rohitash Chandra "Face Detection and Recognition in an Unconstrained Environment for Mobile Visual Assistive System” [2016].

[12] Diajeng Tyas Purwa Hapsari, Cindykia Gusti Berliana, Putri Winda, Dr. M.Arief Soeleman, "Face Detection Using Haar Cascade in Difference Illumination" [2018].

[13] Saikrishna Chatrati, Sumanth Naidu, Ch.Raghava Prasad, "RFID based Student Monitoring and Attendance Tracking System”, July 2013 IEEE.

[14] Fahad-Bin-Mazhar, Oli Ahamed, Md.Rasedujjaman, "Biometric Smart Attendance Kit with Fingerprint Scanner by Using Microcontroller" Nov 2015 IEEE. 